\title{
A Closed-loop Control for Swing System and Energy Regeneration Analyses of the Multi-system of Grasp Steel Machine
}

\section{Yunqi Yan}

Sichuan University of Science \& Engineering

Puquan Wang ( $\sim 13698250260 @ 163 . c o m$ )

Southwest Hospital, Third Military Medical University

\section{Yun Xu}

Sichuan University of Science \& Engineering

Yan Shi

Sichuan University of Science \& Engineering

\section{Yinghua Liao}

Sichuan University of Science \& Engineering

\section{Research Article}

Keywords: grasp steel machine, swing system, CCSS, BASS, OCSS, energy recovery

Posted Date: November 2nd, 2021

DOI: https://doi.org/10.21203/rs.3.rs-993227/v1

License: (c) (i) This work is licensed under a Creative Commons Attribution 4.0 International License. Read Full License 


\title{
A closed-loop control for swing system and energy regeneration analyses of the multi-system of Grasp Steel Machine
}

\author{
Yunqi Yan', Puquan Wang ${ }^{2 *}$, Yun $\mathrm{Xu}^{1}$, Yan Shi ${ }^{1}$ and Yinghua Liao ${ }^{1}$ \\ ${ }^{1}$ Department of Mechanical Engineering, Sichuan University of Science \& Engineering, Huixing Road . No180, \\ Zigong, 643000, China, yyq77mail@163.com, xuyunzigong@163.com, sy71email@163.com, \\ Liaoyinghua118@163.com \\ ${ }^{2}$ Center for Joint Surgery, Southwest Hospital, Third Military Medical University, No. 29 Gaotanyan Street, \\ Shapingba District, Chongqing 400038, China, 13698250260@163.com
}

\begin{abstract}
Facing the environment problems, the improvement on the efficiency of the construction machinery is highly demanded, meanwhile, large energy wasted in hydraulic slewing systems, reaching the desired speed slowly and vibration in braking are widespread. In this paper, a closed-loop control swing system (CCSS) is proposed. In this system, bidirectional variable pump controls the change of oil flow by programmed software algorithm, instead of pilot actuated reversing valve, which significantly improves the efficiency of hydraulic circuit. Based on the model structures, we develop a hydraulic swing prototype and verify the advantages of our design means of theoretical calculation and simulation. It verifies that CCSS and based on accumulator slewing system(BASS) can respectively save $875.5 \mathrm{KJ}$ and 347.2 KJ more than OCSS with operational weight of 100 tons grasp steel machine, which can support theoretical basis for energy conservation and environmental protection to study the new type of engineering machinery.
\end{abstract}

Keywords: grasp steel machine, swing system, CCSS, BASS, OCSS, energy recovery.

\section{Introduction}

Resource shortages and environmental pollution are the increasingly urgent global problems that have encouraged the development of energy-saving [1,2] and emission-reduction technologies [3,4]. It is meaningful to investigate the energy saving of construction machinery, especially for grasp steel machines equipped with hybrid power system due to their wide application while extremely low efficiency [5-8]. The hydraulic grasp steel machine mainly consists of chassis, upper structure and work attachment. The chassis using for a stable base includes crawler and propel drive. Upper structure that is mainly composed of swing machinery and revolving platform is responsible for rotation and transportation. Work attachment being used to perform grab operation consists of boom, arm, crab bucket and associated cylinders, etc. 
Swing motion is one of fundamental motions of a hydraulic grasp steel machine, which accounts for $35 \%$ of the grasp steel machine's total operational time and is driven by hydraulic motors [9]. The characteristics of swing motion are frequent acceleration and deceleration. Sometimes, the problem of the swing motion operation is of extreme roughness and jerkiness, which is mainly related to hydraulic and mechanical properties of the actuators. Mechanical dampers, such as a viscous damper or a coulomb friction damper, are frequently used to reduce vibrations in construction machinery [10]. However, the additional mechanical dampers installed into the swing mechanism may increase cost and the weight of machinery as well as sensitivity [11]. For these reasons, only by altering the hardware mechanism may not be an adequate solution to reduce the swing motor vibration [12].

In 1996, Sepehri firstly studied the gear backlash and stick-slip friction in hydraulic swing motion [13]. In the later time, researchers pay attention to the open-loop control, which is more applicable than closed-loop control algorithm in practical application [14]. The swing inertia of the upper structure was separated into two parts: the calculation and the estimation. The function of calculation is to acquire the position of boom, arm and crab bucket, which can be measured by sensors, and another part is estimated by the payload in the crab bucket. This kind of robust control technique can realize high performance in swing velocity tracking, but it is not applicable for industry. Furthermore, it is difficult to obtain the displacement of cylinder by mounting the sensors[15]. The sliding mode observer and sliding mode control are designed to provide robust tracking performance, which reduce the serious self-exciting vibration of the electric swing motor due to the time-varying swing inertia caused by various working conditions. But the swing motion is discontinuous, it can't satisfy the practical application. Xiao et al. [16] proposed a new hydraulic system for the swing system with a hydraulic accumulator and two flow control valves. This system had some similarities with the presented system in a patent [17]. When the swing system decelerates, the flow rate from the variable displacement hydraulic motor is controlled by the flow control valve and charged to the hydraulic accumulator. Besides, the author was also concerned about the oscillation and energy regeneration efficiency of the proposed system. They designed a control strategy based on a PID controller. Therefore, the proposed system had a much lower cost than the electric ERS system and the energy regeneration efficiency could reach up to $33.4 \%$.

Currently, hybrid technology is regarded as one of the most effective measures to solve the energy waste problem. Heavy vehicles such as grasp steel machines with open-loop control have the characteristics of high stop-and-go duty cycles and high power flow braking energy, which need to find an efficient way to store and reuse the braking energy [18]. In many hybrid power options, lithium-ion battery and fuel cell have the characteristic of high energy density and are well suitable for light vehicles. However, the high internal resistances and handling the wasted battery are major obstacles for commercialization [19]. Both batteries in hybrid vehicles can only marginally recycle the braking energy, and high frequency charging and discharging may lead to overheating and battery destruction $[18,20]$. For another energy recovery component, hydraulic accumulator that has the characteristic of high power density can provide high power for acceleration and also recycle more efficient energy during braking [20]. However, the relatively lower energy density brings the packaging limit about the increase of accumulator size [18]. Another reason for reducing fuel consumption is that the upper structure is driven by the electric swing motor, which is more efficient than 
the hydraulic motors used in conventional hydraulic excavators. Furthermore, the upper structure kinetic energy lost in conventional excavators can be regenerated through deceleration of swing motor [21]. If electric motor is applied to the swing system, there will be a great change in power plant, but it will increase some difficulty in design and implementation of hybrid system.

Aiming at solving the problem of OCSS, this paper presents a closed-loop control algorithmto improve the swing motion performance of grasp steel machine, which are organized as follows: the configuration and principle of all multi-system models are illustrated in section 2, the simulation and analysis is presented in Section 3, and conclusions are drawn in Section4.

\section{The modeling of multisystem}

\subsection{Configuration of the original swing system}

The OCSS has widespread use in slewing system of grasp steel machine because of relatively simple structure. Under the open-loop control mode ,the functions of tank include cooling system oil and precipitating impurities. However, it was the air that easily enters the system, which may lead to inaccurate action of actuator, especially the throttle valve causes great energy losses. In addition, during braking, the mechanical vibration is of extremely sharp, which is easy to generate the hydraulic impact.

As shown in Fig. 1, the constant power variable pump provides power for hydraulic slewing system. Rotating handles control the direction of rotation and take part in adjusting the revolving speed of rotary motor. The pressure oil flows through multi-channel valve 5 to the motor 7 , and then the pressure difference between inlet and outlet of motor will drive rotary motor to work. The main relief valve 10 ensures that the operating pressure does not exceed the line relief pressure and it improves the efficiency of the circuit. The movement of motor is depended on the oil that open the electromagnetic directional valve in gear pump 2 when the motor is in start-up and braking. The overflow valve can control the starting and braking pressure and check valve can fill oil in time. If slewing platform turns too fast, check valve 9 can fill oil into the motor. The pilot control valve 4 is essentially a straight moving type relief valve which can release outlet pressure by adjusting control handle.

The model of slewing system of grasp steel machine is built in the AMESim, Which can be shown in Fig. 2. 


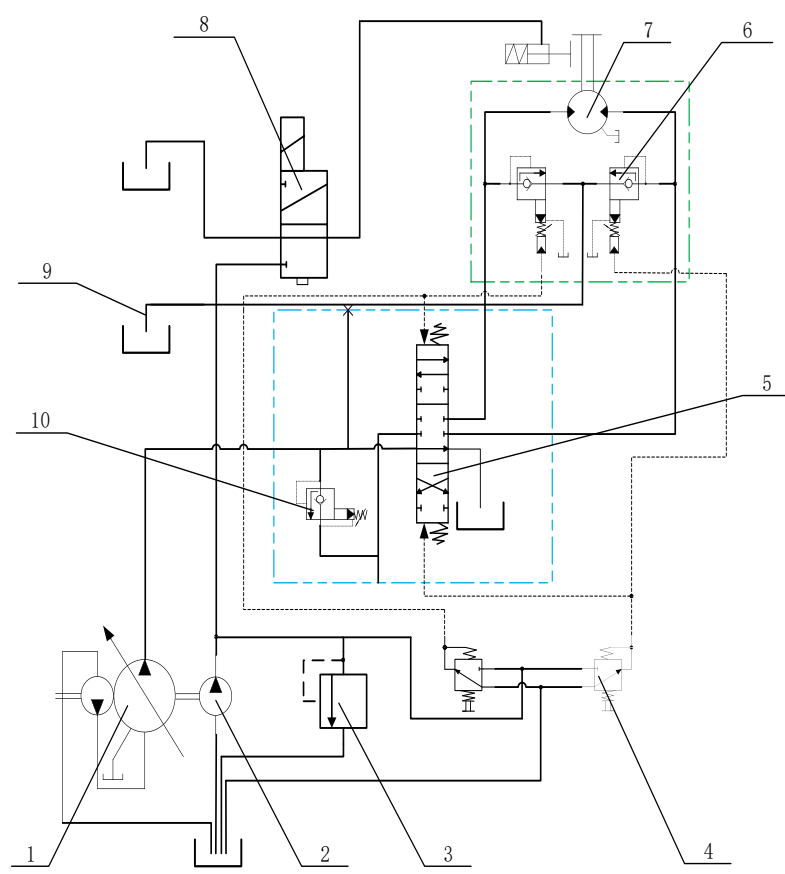

Fig. 1 Slewing system of WZY70-7 grasp steel machine

1 - variable pump; 2 - the forerunner gear pump; 3 - pilot relief valve;

4 - pilot control valve;5 - multi-way valve;6 - rotary relief valve;

7 - motor;8 - electromagnetic directional valve;9 - check valve; 10 - the main relief valve

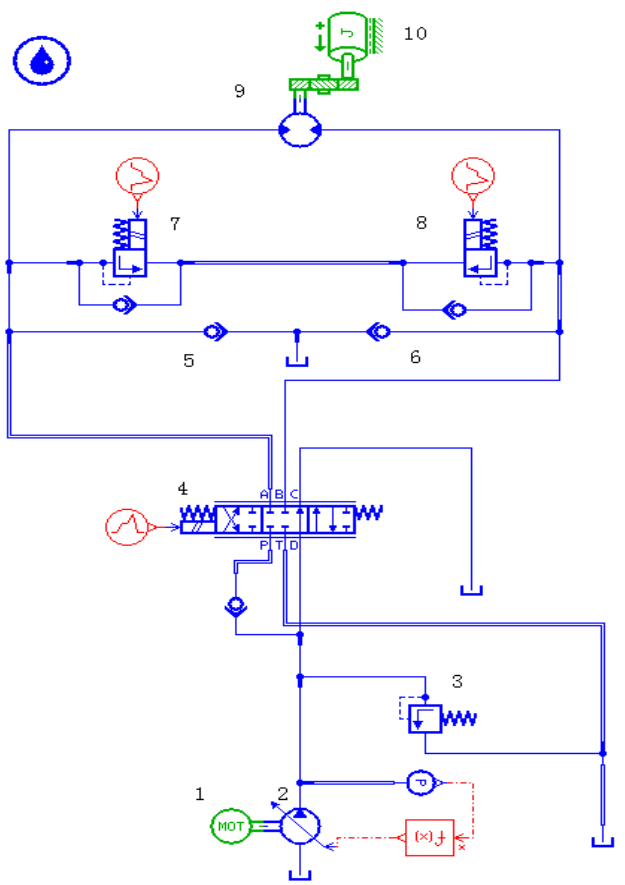

Fig. 2 Simulation model of slewing system of grasp steel machine

1 - motor;2 - variable pump;3 - the overflow valve; 5, 6 - check valve; 4 - pilot actuated reversing valve;

7, 8 - reducing valve;9 - motor; 10 - load

\subsection{The modeling of based on accumulator slewing system}

A new system based on the slewing system of WZY70-7 grasp steel machine consists of a hydraulic accumulator and other components, which is BASS as shown in Fig. 3. When pilot actuated reversing valve 2 applies to the left position, slewing platform turns forward. One part of oil that comes from pump flows through pilot actuated reversing valve 2 to drive motor 6 , and another part of oil is stored in the accumulator 5 by directional control valve 4 and hydraulic controlled check valve 3 . As speeding up slewing platform, the oil absorbed by rotary motor increasing. When the oil flow is equal to oil supplied by pump, rotation of motor keeps constant.

When the pilot actuated reversing valve 2 turns to middle position, the oil driving rotary motor is cut off, but the slewing platform continues rolling under the inertia effect. During the braking operations, the swing motor functions as a pump and drives accumulator which, in turn, acts as a hydraulic motor. In other words, it realizes the braking energy recovery so that the oil can store in the accumulator 5 by directional control valve 4 and hydraulic controlled check valve 3 . When pilot control reversing valve 2 applies to the right position, slewing platform reverse rotation. Combining the oil stored in accumulator during overflow recycling and braking with pump to drive rotary motor, which reduces the outlet power of the pump. 


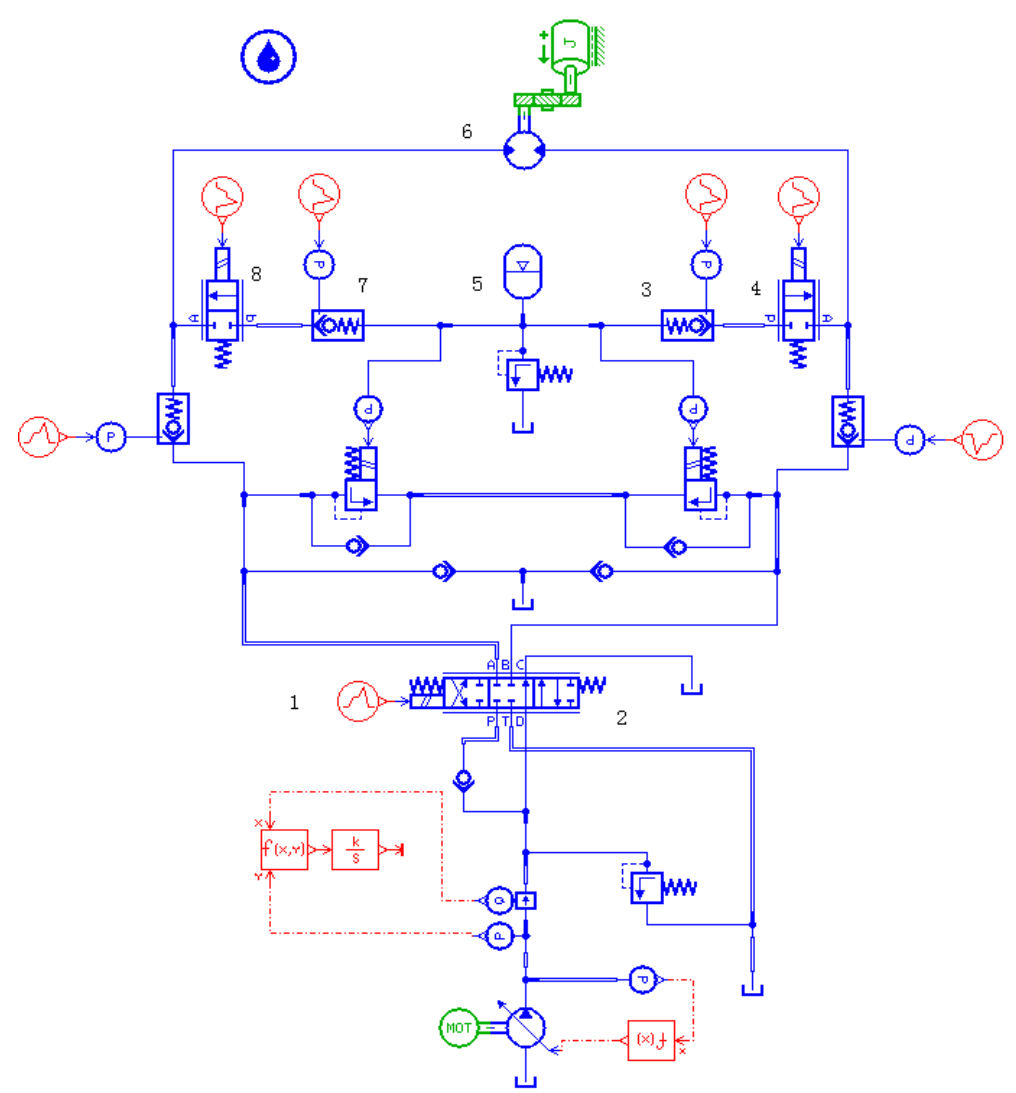

Fig. 3 Simulation model based on the accumulator swing system

1 -Control signal; 2- pilot actuated reversing valve; 3,7- hydraulic controlled check valve; 4,8- directional control valve;

5- accumulator; 6- motor

\subsection{Modeling of closed-loop control slewing system}

In short, the function of CCSS is that a hydraulic pump drives hydraulic motor. Compared with OCSS, the reversal, forward and braking can't be realized by the valve. The rotation direction of swing motors is determined by the directional function of swing pump, and the rotation speed is proportional to the amount of swing pump's volumetric displacement. When the variable displacement pump is positioned in a neutral position, there is no volumetric fluid flowing to the swing motor. Meanwhile, the turning of motor is controlled by the direction of the swash plate of variable pump, the speed of motor is controlled by the angle of swash plate of variable pump. In a word, the variable pump and variable motor are important. It is inevitable to encounter the problem of huge rotational inertia and inaccurate angle when large is turning. Therefore, applying the CCSS to slewing mechanism can realize stepless speed regulation in the braking and starting processes, which can reduce impact and achieve energy recovery.

The model of CCSS is depicted in Fig. 4. The bidirectional variable pump 2[22] and quantitative pump 10 are controlled by a variable motor 1 . The leakage and pressure of whole system are decided by quantitative pump 10 and overflow valve 7. A quantitative pump 10 supplies oil through piloted pressure reducing valve and check valve for CCSS system to keep the pressure in each circuit, which can prevent cavitations. The pump control behavior is independent of external operating parameters due to the fact that signal symbol substitutes variable displacement control mechanism in constant power pump, which is diverged from the charging pump flow through a piloted pressure reducing valve. If there is a large load impact, the balancing valve 3 and 4 will protect pressure equilibrium. Electromagnetic directional valve 9 is free to control the motor on and off. At the end of braking, 
inertial kinetic energy will make hydraulic motor reversal, which causes vibration and noise. At the same time, big resilience force leads to backswing for many times, and it may cause shock. For this kind of situation, the defensive valve 9 can prevent rebounding in hydraulic rotary system and make hydraulic motor switch on or shut down. In a word, this configuration allows a highly dynamic and precise control of the pump delivery flow rate according to the input current demand. Thus, it not only reduces the system losses, but also easily and accurately regulates the flow to the swing motor as well as the rotation speed of the motor. During the braking operation, the upper structure of hydraulic shovel tends to continue to rotate until it is fully stopped due to the huge swing inertia of moving components. It is noteworthy that the setting of the time-varying swing inertia requires special processing, which can be done through analog signals and signal feedback. To sum up, compared with existing system, the proposed CCSS for swing motion of hydraulic is simpler as it is controlled by control device instead of manual adjustment system. In the next, we will demonstrate new system's advantages through simulation on a prototype swing unit of a hydraulic grasp steel machine whose operational weight is 100 tons.

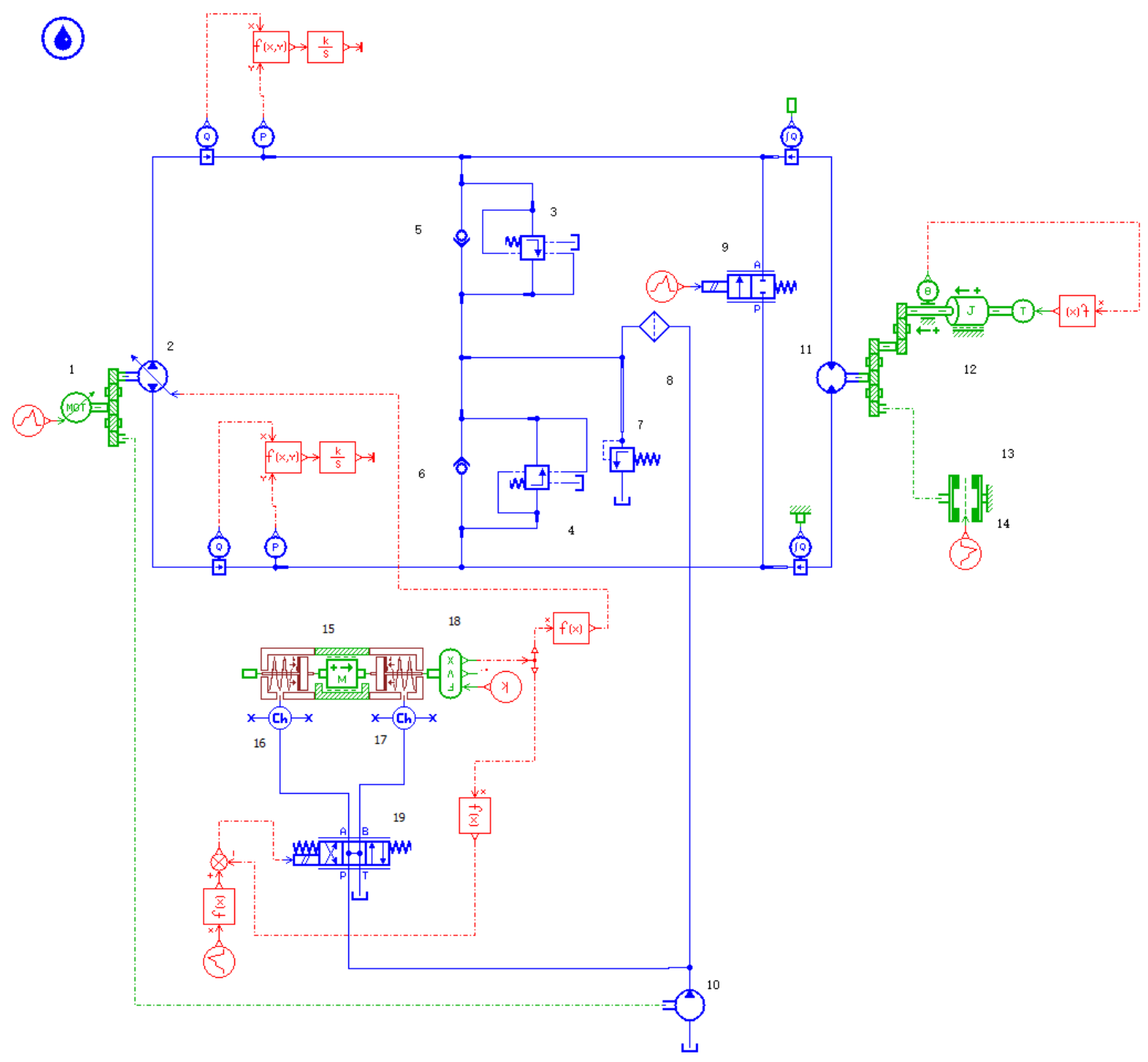

Fig. 4 Simulation model of closed-loop control slewing system

1 - variable motor; - the bidirectional variable pump;3, 4 - balancing valve;5, 6 - check valve; 7 - the overflow valve; 8 - filter; 9 - defensive valve;10 - quantitative pump;11 - motor;12 - mechanical gear reducer;13 - load;14 - brake;15-

\section{Mathematical models}


In the CCSS, the change of the variable in the variable pump depends on the swash plate swing angle of the pump which is changed by a hydraulic cylinder controlled by a proportional valve or a servo valve.

Compared with the slewing transmission mechanism of the steel grabber, the dependent variable mechanism not only has a small inertia, but also has a fast response speed. Therefore, the following derivation is a mathematical model from the swash plate swing angle of the variable pump to the motor output angle.

In order to facilitate the establishment of the modeling of closed-loop control slewing system, we make the following assumptions. The speed of the pump is constant, the leakage of the pump and the hydraulic motor is laminar flow, the oil return pressure of the casing is atmospheric pressure, and the leakage of the low pressure cavity into the casing is ignored. Because the connecting pipeline is hard and short, the pressure loss, fluid mass effect and dynamic changes in the pipeline can be ignored. The two pipes are exactly the same, the total volume of the two chambers composed of the pump, the motor and the pipe are equal, the temperature and volume elastic modulus of the oil in each chamber are constant, and the pressure is uniform and equal. There is no hysteresis in the pressure and flow of the replenishment system, ignoring the influence of instantaneous load changes. It is considered that the refueling pressure is constant, that is the low cavity pressure during work, and only the high pressure cavity pressure changes. Because the rigidity of the connection structure between the hydraulic motor and the load is very large, the influence of the structural flexibility is ignored. The input signal is small, no pressure saturation occurs. There is no pressure shock in the pipeline, and its pressure does not exceed the pressure of the safety valve

The displacement equation of the variable pump is calculated as follow.

$$
D_{p}=k_{p} r
$$

where $k_{p}$ is the displacement gradient of variable pump, $r$ is the swing angle of variable pump variable mechanism.

The flow equation of the variable pump is calculated as follow.

$$
Q_{p}=D_{p} \omega_{p}-C_{i p}\left(p_{1}-p_{r}\right)-C_{e p} p_{1}
$$

where $Q_{P}$ is the output flow of variable pump, $\omega_{p}$ is the angular velocity of variable pump, $C_{i p}$ is the internal leakage coefficient of pump, $P_{1}$ is the pressure of high pressure pipeline, $P_{\mathrm{r}}$ is the charge pressure of low pressure pipeline and assumed to be a constant. $C_{e p}$ is the leakage coefficient of pump.

Since $P_{\mathrm{r}}$ is a constant, by substituting Eq. (1) into Eq. (2), the incremental equation laplace transform of the 
flow equation can be rewritten as:

$$
Q_{p}=K_{q p} r-C_{t p} P_{1}
$$

Where $K_{q p}$ is the flow gain of variable pump $\left(K_{q p}=k_{p} \omega_{p}\right) . C_{t p}$ is the total leakage factor of the pump which equal to $C_{i p}$ plus $C_{e p}$.

Assuming that all the output flow of the variable pump enters the motor, the flow continuity equation of the high pressure chamber of the hydraulic motor is derived as:

$$
Q_{p}=C_{i m}\left(p_{1}-p_{r}\right)+C_{e m} p_{1}+D_{m} \frac{d \theta_{m}}{d t}+\frac{V_{0}}{\beta_{e}} \frac{d p_{1}}{d t}
$$

Where $C_{i m}$ is the leakage coefficient in hydraulic motor, $C_{e m}$ is the leakage coefficient of hydraulic motor, $D_{m}$ is the displacement of hydraulic motor, $\theta_{m}$ is the shaft angle of hydraulic motor, $\beta_{e}$ is the effective bulk modulus of elasticity including mechanical flexibility of the cylinder, oil and connecting pipes, $V_{0}$ is the total volume of a chamber including a working chamber of pump and hydraulic motor, connecting pipe and non-working volume connected to them. The pull transformation of the incremental equation can be rewritten as:

$$
Q_{p}=C_{t m} P_{1}+D_{m} s \theta_{m}+\frac{V_{0}}{\beta_{e}} s P_{1}
$$

Where $C_{t m}$ is the total leakage coefficient of hydraulic motor, which equal to $C_{i m}$ plus $C_{e m}$.

The torque balance equation of motor and load can be described as:

$$
D_{m}\left(p_{1}-p_{r}\right)=J_{t} \frac{d^{2} \theta_{m}}{d t^{2}}+B_{m} \frac{d \theta_{m}}{d t}+G \theta_{m}+T_{L}
$$

Where $J_{t}$ is the total inertia of motor and load that is converted to the motor shaft, $B_{m}$ is the total viscous damping coefficient of the motor and the load converted to the motor shaft. $G$ is the load stiffness, $T_{L}$ is the any external load torque acting on the motor shaft. Its pull transform of incremental equation can be rewritten as:

$$
D_{m} P_{1}=J_{t} s \theta_{m}+B_{m} s \theta_{m}+G \theta_{m}+T_{L}
$$


The Eqs. (3), (5), and (7) are the three basic equations of the closed hydraulic system by pump-controlled motor and fully describe the dynamic characteristics of it.

The transfer function of the closed hydraulic system by pump-controlled motor can be obtained from the Eqs. (3), (5), and (7) as follow:

$$
\theta_{m}=\frac{\frac{K_{p q}}{D_{m}} \gamma-\frac{C_{t}}{D_{m}^{2}}\left(1+\frac{V_{0}}{\beta_{e} C_{t}} s\right) T_{L}}{\frac{V_{0} J_{t}}{\beta_{e} D_{m}^{2}} s^{3}+\left(\frac{C_{t} J_{t}}{D_{m}^{2}}+\frac{B_{m} V_{0}}{\beta_{e} D_{m}^{2}}\right) s^{2}+\left(1+\frac{B_{m} C_{t}}{D_{m}^{2}}+\frac{G V_{0}}{\beta_{e} D_{m}^{2}}\right) s+\frac{G C_{t}}{D_{m}^{2}}}
$$

Where $C_{t}$ is the total leakage factor and equal to $C_{t}$ plus $C_{t}$. In Eq. (8), the factors such as inertial load, viscous friction load, elastic load and oil compression and motor leakage are considered. But the actual system load is often relatively simple, and some influencing factors can be ignored according to the specific usage. The load of the servo system is dominated by inertial load in many cases, and there is no elastic load or the elastic load is very small and can be ignored. In general, there is no elastic load, hydraulic motors act as actuators in Servo system. Therefore, it is assumed that there is no elastic $\operatorname{load}(G=0)$.

In addition, the $B_{m}$ is very small, the $D_{m}{ }^{2} / C_{t}$ is much larger than $B_{m}$. The Eq. (8) can be simplified as:

$$
\theta_{m}=\frac{\frac{K_{q p}}{D_{m}} \gamma-\frac{C_{t}}{D_{m}^{2}}\left(1+\frac{V_{0}}{\beta_{e} C_{t}} s\right) T_{L}}{s\left(\frac{s^{2}}{\omega_{h}^{2}}+\frac{2 \zeta_{h}}{\omega_{h}} s+1\right)}
$$

Where $\omega_{h}$ is the hydraulic natural frequency, it described as:

$$
\omega_{h}=\sqrt{\frac{\beta_{e} D_{m}^{2}}{V_{0} J_{t}}}
$$

Where $\zeta_{h}$ is the hydraulic damping ratio and described as:

$$
\zeta_{h}=\frac{C_{t}}{2 D_{m}} \sqrt{\frac{\beta_{e} J}{V_{0}}}+\frac{B_{m}}{2 D_{m}} \sqrt{\frac{V_{0}}{\beta_{e} J_{t}}}
$$

By substituting Eqs. (10) and (11) for the according variables in Eq. (12), the transfer function of the output shaft angle of the hydraulic motor to the swash plate swing angle of the variable pump can be rewritten as: 


$$
\frac{\theta_{m}}{\gamma}=\frac{\frac{K_{q p}}{D_{m}}}{s\left(\frac{s^{2}}{\omega_{h}^{2}}+\frac{2 \zeta_{h}}{\omega_{h}} s+1\right)}
$$

And then, Eq. (12) can be rewritten as:

$$
\frac{\theta_{m}(s)}{\gamma(s)}=\frac{\frac{K_{q p}}{D_{m}}}{\frac{s^{2}}{\omega_{h}^{2}}+\frac{2 \zeta_{h}}{\omega_{h}} s+1}
$$

The transfer function of the hydraulic motor shaft angle to any external load torque, and also it's the dynamic flexibility of the system can be described as:

$$
\frac{\theta_{m}}{T_{L}}=\frac{-\frac{C_{t}}{D_{m}^{2}}\left(1+\frac{V_{0}}{\beta_{e} C_{t}} s\right)}{s\left(\frac{s^{2}}{\omega_{h}^{2}}+\frac{2 \zeta_{h}}{\omega_{h}} s+1\right)}
$$

And then, Eq. (14) can be rewritten as:

$$
\frac{\theta_{m}(s)}{T_{L}(s)}=\frac{-\frac{C_{t}}{D_{m}^{2}}\left(1+\frac{V_{0}}{\beta_{e} C_{t}} s\right)}{\frac{s^{2}}{\omega_{h}^{2}}+\frac{2 \zeta_{h}}{\omega_{h}} s+1}
$$

\section{Simulation}

\subsection{Parameter Setting}

1. Moment of resistance in rotary

(1) Wind resistance torque

$M_{\text {huifbi }}$ is the rotational resistance torque on arm and bucket caused by wind, which is defined as follows:

$$
\begin{gathered}
F_{\text {huifbi }}=q_{1} \times C_{b i} \times j(1+h) \times L_{b i h} \times L \\
M_{\text {huifbi }}=F_{\text {huifbi }} \times X_{b i}
\end{gathered}
$$

Where $F_{h u}$ ifbi is wind load of arm and bucket rod, $C_{b i}$ is wind power coefficient, if $q_{1} \times D^{2}<3, C_{b i}=1.2, D$ is chord diameter. The structure of the filling percentage, $\varphi=0.3$, Wind reduction factor, $\eta=0.575, L_{b i h}$ is the height of cross section of movable arm and bucket rod, $L$ is arm length, $X_{b i}$ is the center of gravity position.

$M_{\text {huitai }}$ is the rotational resistance torque on slewing platform caused by wind, which is defined as follows: 


$$
\begin{gathered}
F_{\text {huifai }}=C \times q_{1} \times S_{\text {tai }} \\
M_{\text {huifai }}=F_{\text {huifai }} \times X_{t a i}
\end{gathered}
$$

Where $F_{\text {huifai }}$ is wind load, $C$ is wind power coefficient, $C=1.2, S_{\text {tai }}$ is turntable lateral windward area, $X_{\text {tai }}$ is turntable lateral centroid position.

$M_{\text {huifq }}$ is the moment of resistance caused by wind, which is defined as follows:

$$
\begin{aligned}
& F_{\text {huifq }}=C \times q_{1} \times S_{q} \\
& M_{\text {huifq }}=F_{\text {huifq }} \times R
\end{aligned}
$$

Where $F_{\text {huifq }}$ is wind load, $C$ is wind power coefficient, $C=1.3, S_{q}$ is load windward area, $R$ is amplitude.

The whole rotational resistance torque is $M_{\text {huif }}$

$$
M_{\text {huif }}=M_{\text {huifbi }}+M_{\text {huiftai }}+M_{\text {huifq }}
$$

(2) Friction resistance moment

$$
\begin{gathered}
F_{\text {huim }}=F_{\text {hui }}\left(1-\frac{2 \varphi}{\pi}\right)+\frac{2 K \times M_{\text {hui }} \times \sin \varphi}{\pi D} \\
M_{\text {huizm }}=0.5 f_{\text {hui }} \times F_{\text {huim }} \times D_{\text {hui }}
\end{gathered}
$$

Where $\varphi$ is the angle between the raceway and the level surface, $-\varphi=\cos ^{-1}\left(\frac{D h u i \times F h u i}{K \times M u i}\right), K$ is a factor, considering a axial clearance, the number of bearing roller will reduce, thus the maximum load on rolling element bearing will increase, $K=4, F_{h u i}$ is rolling friction coefficient, $f_{\text {hui }}=0.0025, D_{\text {hui }}$ is roller diameter.

(3) Inertia resistance moment

$$
\begin{gathered}
J_{h u i}=Q \times R^{2}+G_{p e i} \times X_{p e i}{ }^{2}+G_{t a i} \times X_{t a i}{ }^{2}+G_{b i} \times X_{b i}{ }^{2} \\
E_{h u i}=\frac{2 \pi \times V_{h u i}}{60 \times t_{q}} \\
M_{\text {huig }}=1.5 J_{h u i} \times E_{h u i}
\end{gathered}
$$

Where $J_{h u i}$ is the moment of inertia, $V_{h u i}$ is rotary speed under full load, $t_{q}$ is starting time, $E_{h u i}$ is angular acceleration.

Normal resistance moment can be rewritten as:

$$
M_{\text {huizong }}=M_{\text {huif }}+M_{\text {huim }}
$$

Maximum resistance moment can be rewritten as:

$$
M_{\text {huizong }}=M_{\text {huif }}+M_{\text {huip }}+M_{\text {huig }}
$$

According to formula above to process calculating and decide which version of the weight of 100 tons grasp steel machine to choose. The most unfavorable condition of rotary mechanism is that both rotating and grabbing happen simultaneously. In addition, actuator still needs to grab something in maximum weight. The related parameters can be shown in Table 1. 
Table 1 Parameters under the most unfavorable condition

\begin{tabular}{ccccc}
\hline $\begin{array}{c}\text { Wind resistance } \\
\text { torque }(\mathrm{kNm})\end{array}$ & $\begin{array}{c}\text { Friction resistance } \\
\text { moment }(\mathrm{kNm})\end{array}$ & $\begin{array}{c}\text { Inertia resistance } \\
\text { moment }(\mathrm{kNm})\end{array}$ & $\begin{array}{c}\text { Normal } \\
\text { resistance } \\
\text { moment }(\mathrm{kNm})\end{array}$ & $\begin{array}{c}\text { Maximum } \\
\text { resistance } \\
\text { moment } \\
(\mathrm{kNm})\end{array}$ \\
\hline 8.78 & 16.60 & 70.37 & 249.61 & 312.43 \\
\hline
\end{tabular}

\section{Component parameters}

Through the calculation on model of grasp steel machine and above calculation results of resistance torque, it can be obtained that the full load moment of inertia of rotation is $907800 \mathrm{~kg} \bullet \mathrm{m}^{2}$ and no-load is $506334 \mathrm{~kg} \bullet \mathrm{m}^{2}$.

$$
\begin{gathered}
T=\left(\mathrm{t}_{Q}+\mathrm{t}_{Q}^{o}\right)+\left(\mathrm{t}_{y}+\mathrm{t}_{y}^{o}\right)+\left(\mathrm{t}_{z}+\mathrm{t}_{z}^{o}\right)=\frac{J_{o}(1+\lambda) \omega_{Q}}{2 K M_{Q}} \times\left(1+\frac{1}{C}\right)+\frac{2 \phi}{\omega_{Q}}(\mathrm{~s}) \\
M_{Q}=\frac{1000 N \eta_{Q}}{K \omega_{Q}}
\end{gathered}
$$

Where $t_{Q}$ is starting time of full bucket on turntable, $\mathrm{t}^{\mathrm{o}} \mathrm{Q}$ is starting time of empty bucket on turntable, $\mathrm{t}_{\mathrm{y}}$ and $\mathrm{t}_{\mathrm{y}}^{\mathrm{o}}$ are rotation times in uniform speed with full and empty bucket, $\mathrm{t}_{\mathrm{z}}$ and $\mathrm{t}_{\mathrm{z}}^{\mathrm{o}}$ are rotation times in braking with full and empty bucket, $\omega_{Q}$ is rotary angular velocity, $M_{Q}$ is rotary starting torque, $\phi$ is corner, $C$ is coefficient, $C=1.361, \lambda=J / J_{o}, J_{o}$ and $J$ are rotary inertias of empty and full bucket, $K$ is related with the friction coefficient and the value is $0.87 \sim 0.92 . N$ is hydraulic power of slewing mechanism, $\eta_{o}$ is total efficiency of slewing mechanism. Combining Eq. (15) with Eq. (16) to conclude:

$$
T=\frac{J_{\mathrm{o}}(1+\lambda) \omega_{Q}^{2}}{2000 N \eta_{o}} \times\left(1+\frac{1}{C}\right)+\frac{2 \phi}{\omega_{Q}}
$$

The formula of best speed of the slewing platform comes from $\frac{\mathrm{d} T}{d \omega_{Q}}=0, \frac{d^{2} T}{d^{2} \omega_{Q}}>0$ :

$$
\omega_{Q}=\omega_{\max }=\sqrt[3]{\frac{2000 N \eta_{o} \phi}{J_{o}(1+\lambda)\left(1+\frac{1}{C}\right)}}
$$

The highest torque of rotary motor is

$$
M=\frac{\mathrm{p} \times q \times \eta_{m}^{N}}{6.28}=\frac{26 \times 160 \times 0.97}{6.28}=643(\mathrm{~N} \cdot \mathrm{m})
$$

Where $P$ is the pressure of motor, rotary overload pressure is $26 / 12 \mathrm{Mpa}, \eta_{m}^{N}$ is volume efficiency of the value is 0.97 .

The version of rotary deceleration machine is GFB110T3, speed ratio $i_{1}=80.52$, the outlet gear $Z_{1}=13$. The version of rotary rolling plate is $134.45 .2139, \mathrm{Z}_{2}=82$, the speed ratio $\mathrm{i}_{2}=82 / 13=6.31$.

Maximum speed of slewing platform can be obtained as follows: 


$$
\begin{gathered}
\mathrm{n}_{\text {max }}=\frac{n_{\text {max }}}{i_{1} \times i_{2}}=\frac{3872}{80.52 \times 6.31}=7.6(\mathrm{r} / \mathrm{min}) \\
\omega_{\max }=\frac{n_{\text {max }} \times 2 \pi}{60}=\frac{7.6 \times 2 \pi}{60}=0.795(\mathrm{rad} / \mathrm{s})
\end{gathered}
$$

The requirement of choosing appropriate accumulator can be listed as follow:

In a working period, the accumulator collects all the overflow and braking energy as much as possible. At the same time, releasing the absorbed energy to achieve a high energy recycling efficiency. Meanwhile, due to the limitations of the layout space of grasp steel machine, accumulator with light weight and small volume should be chosen as far as possible.

$P_{2}$ is determined by the peak pressure of the whole system, and it is $26.4 \mathrm{MPa}$. As auxiliary power supply, $P_{l}$ is generally equal to $0.6 \sim 0.85 P_{2}$ in accumulator; Considering extending the service life of accumulator, $P_{2} \leqslant 3 P_{1}$. Combining above conditions, $P_{1}$ is 15 20MPa.

$$
E=\int_{V_{1 a}}^{V_{2 a}} p d V=-\int_{V_{1 a}}^{V_{2 a}}\left(\frac{V_{0}}{V}\right)^{1.4} d V=\frac{P_{0} V_{0}}{0.4}\left[\left(\frac{P_{2 a}}{P_{0}}\right)^{0.286}-\left(\frac{P_{1 a}}{P_{0}}\right)^{0.286}\right]
$$

According to the above formula, when $P_{1 a}$ is $P_{0}, P_{2 a}$ is $P_{2}$ and the maximum absorbed energy of hydraulic accumulator is $\mathrm{E}_{\max }$, $P_{0}$ is $20 \mathrm{MPa}$.

$$
\begin{gathered}
P_{0} V_{0}^{n}=P_{1} V_{1}^{n}=P_{2} V_{2}^{n}=\text { const } \\
V_{0}=\frac{V_{w}}{P_{0}^{\frac{1}{n}}\left[\left(\frac{1}{P_{1}}\right)^{\frac{1}{n}}-\left(\frac{1}{P_{2}}\right)^{\frac{1}{n}}\right]}=\frac{V_{1}-V_{2}}{8^{0.714}\left(0.05^{0.714}-0.038^{0.714}\right)}=\frac{V_{1}-V_{2}}{0.0925}
\end{gathered}
$$

\begin{tabular}{|c|c|c|c|c|}
\hline Variable pump & $\begin{array}{c}\text { Displacement/ } \\
(\mathrm{mL} \cdot \mathrm{r}-1) \\
260\end{array}$ & $\begin{array}{l}\text { Rated speed/ } \\
(\mathrm{r} \cdot \mathrm{min}-1) \\
2200\end{array}$ & $\begin{array}{c}\text { Mechanical } \\
\text { efficiency } \\
0.9\end{array}$ & $\begin{array}{c}\text { Volumetric } \\
\text { efficiency } \\
0.97\end{array}$ \\
\hline $\begin{array}{c}\text { Directional } \\
\text { control valve }\end{array}$ & $\begin{array}{l}\text { Rated flow/ } \\
(\mathrm{L} \cdot \min -1)\end{array}$ & $\begin{array}{c}\text { The rated } \\
\text { pressure } \\
\text { differential/ MPa }\end{array}$ & Rated current/mA & Frequency/Hz \\
\hline & 500 & 1 & 40 & 80 \\
\hline $\begin{array}{l}\text { Quantitative } \\
\text { pump }\end{array}$ & $\begin{array}{c}\text { Displacement/ } \\
(\mathrm{mL} \cdot \mathrm{r}-1) \\
30\end{array}$ & $\begin{array}{c}\text { Rated speed/ } \\
(\mathrm{r} \cdot \min -1) \\
1000\end{array}$ & $\begin{array}{c}\text { Mechanical } \\
\text { efficiency } \\
0.92\end{array}$ & $\begin{array}{c}\text { Volumetric } \\
\text { efficiency } \\
0.97\end{array}$ \\
\hline Hydraulic motor & $\begin{array}{c}\text { Displacement/ } \\
(\mathrm{mL} \cdot \mathrm{r}-1) \\
125\end{array}$ & $\begin{array}{c}\text { Rated speed/ } \\
(r \cdot \min -1) \\
2200\end{array}$ & $\begin{array}{c}\text { Mechanical } \\
\text { efficiency } \\
0.9\end{array}$ & $\begin{array}{c}\text { Volumetric } \\
\text { efficiency } \\
0.97\end{array}$ \\
\hline Accumulator & $\begin{array}{c}\text { volume/L } \\
18\end{array}$ & $\begin{array}{l}\text { Highest working } \\
\text { pressure/ } \mathrm{MPa} \\
26\end{array}$ & $\begin{array}{c}\text { Minimum } \\
\text { working pressure/ } \\
\mathrm{MPa} \\
18\end{array}$ & $\begin{array}{c}\text { Inflation pressure/ } \\
\mathrm{MPa} \\
20\end{array}$ \\
\hline
\end{tabular}

Through calculation, the $V_{0}$ is $18 \mathrm{~L}$.

The parameters of the main hydraulic components in mode can be shown shown in Table 2.

Table 2 Parameters of the proposed model 


\begin{tabular}{|c|c|c|c|}
\hline The relief valve & $\begin{array}{l}\text { The rated } \\
\text { pressure(open } \\
\text { system)/MPa }\end{array}$ & $\begin{array}{c}\text { The rated } \\
\text { pressure(closed } \\
\text { system)/MPa }\end{array}$ & Pressure of valve fully open/MPa \\
\hline & 35 & 3 & 26 \\
\hline
\end{tabular}

\subsection{Simulation and analysis}

According to the measured operational data of grasp steel machine, setting up the simulation cycle of $45 \mathrm{~s}$ and taking the motion of upper structure as a typical work cycle: forward, braking and reverse, which contributes to acquiring true analog load of excavator. Main parameters of hydraulic system are shown as follows: forward time is 20 s, reverse time is 20 s, braking time is 5 s for the OCSS.

According to the setting parameters above, obtaining speed curve of the motor. As shown in Fig. 5, the red curve of motor speed seems to "double trapezoid" in OCSS. When reversing valve in the left position, the motor accelerates forward from $0 \mathrm{~s}$ to 7 s, movement at a constant speed from $7 \mathrm{~s}$ to $15 \mathrm{~s}$, slowdown forward from $15 \mathrm{~s}$ to $20 \mathrm{~s}$; When the reversing valve in the right, the motor accelerates reverse rotation from $26 \mathrm{~s}$ to $32 \mathrm{~s}$, movement at a constant speed from $32 \mathrm{~s}$ to $40 \mathrm{~s}$, slowdown reverse rotation from $40 \mathrm{~s}$ to $45 \mathrm{~s}$. For closed hydraulic rotary system, the motor speed curve presents "double rectangle", which turns at a constant speed from $0 \mathrm{~s}$ to $20 \mathrm{~s}$, and turns reversal at a constant speed from $20 \mathrm{~s}$ to $40 \mathrm{~s}$.

The response is rapid in CCSS, the upper structure can turn to cargo location fast, then catch and unload accurately. The driver is easy to operate and improve the efficiency greatly. When grasp steel machines are used to carry and dump oil, sand and other materials in mines, quarries, and construction sites, the swing operation of upper structure is quite frequent, acceleration and deceleration time is quite short. So it is required that the speed control system should have quick speed response and avoid motor torque jerk and speed overshoot to ensure the driver's comfortability [23].

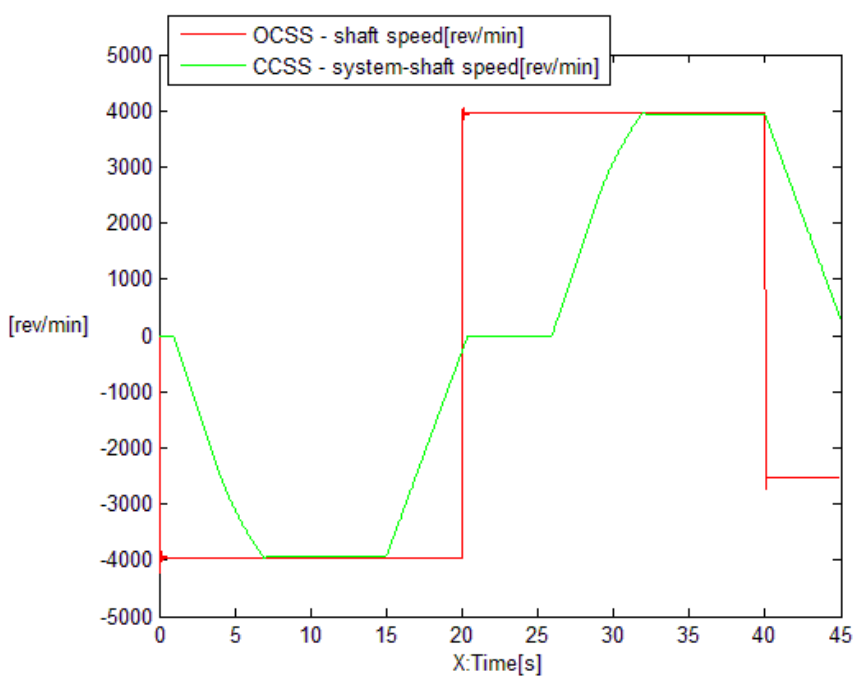

Fig. 5 Speed characteristic curve of motor

As shown in Fig. 6, the red curve is the inlet pressure of pump in OCSS. When the time from 0 to $7 \mathrm{~s}$, the motor is in forward condition and the inlet pressure of pump is 264 bar. When the time from 7 s to $15 \mathrm{~s}$, movement at a constant speed, the inlet pressure drops to 63 bar. The pressure curve in motor reversal is the same as forward. The larger changes of pump inlet pressure 
affect hydraulic components and cause higher calorific value, which may reduce service life of rotary system. However, the maximum differential pressure of pump in closed system is 261 bar and then it will keep at 15 bar after $2.5 \mathrm{~s}$.

In general, the maximum pressure differential that is generated on both outlets of pump in CCSS is 256 bar, which is smaller than the pressure of pump in OCSS. The duration time of maximum pressure difference is 4.1s, that is much shorter than OCSS. At the duration time, the minimum pressure value is 54 bar. Furthermore, there is an obvious jitter from 7.1 to 8.6 s in the OCSS. From the above analysis, we can conclude that small hydraulic components in CCSS withstand less impact and produce less heat, which benefit to prolong the life of the whole system, furthermore, making the rotary motion more smooth.

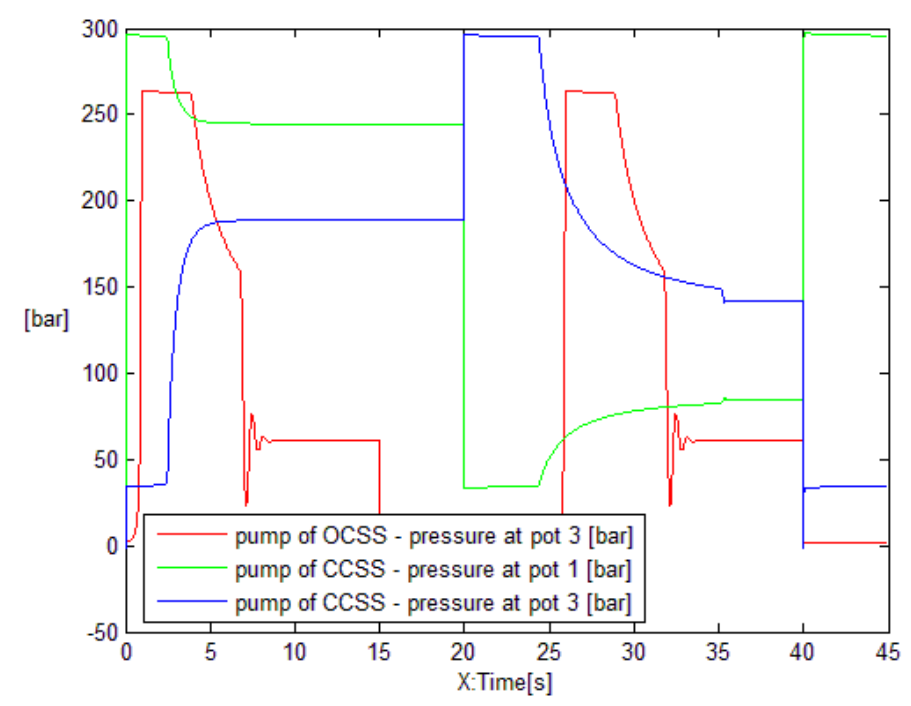

Fig. 6 Pressure characteristic curve of variable pump

As shown in Fig. 7, the inlet and outlet pressure curve of motor in OCSS is obvious jitter, which causes unstable movement of slewing platform in the 7.1-8.6s and 32.2-34.3s. This kind of situation will have impact on stability of hydraulic components and system. However, in the CCSS, the duration of maximum pressure differential of the motor is $4.7 \mathrm{~s}$. Comparing with the $5.3 \mathrm{~s}$ in the OCSS, the motor has a more efficient response. In addition, as the direction of the motor in the OCSS is controlled by the reversing valve, the characteristic combination curve shows that the braking process keeps for 10s or so. In a word, the pressure curve of motor is smooth. Without an obvious dithering phenomenon, hydraulic components receive slight impact and produce less heat, which contributes to extend service life of the whole system. At the same time, the stability of turning motion is improved obviously. 


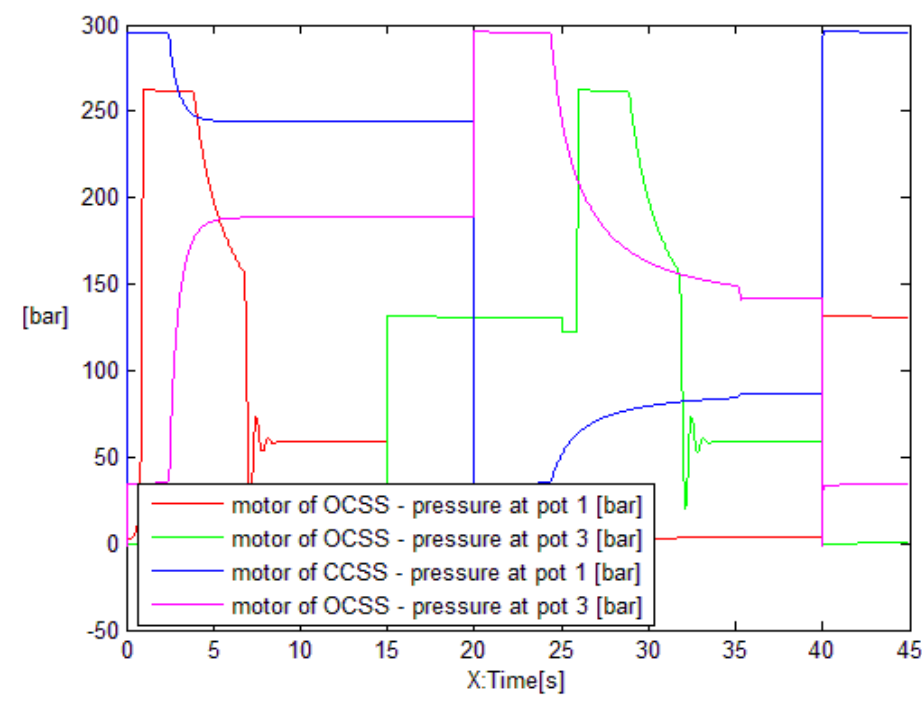

Fig. 7 Pressure characteristic curve of variable motor

Putting the identified accumulator parameters into rotary energy-saving model and processing simulation, it will obtain the characteristic curve of gas volume and gas pressure of accumulator as shown in Fig. 8. During the time from $0 \mathrm{~s}$ to $6.28 \mathrm{~s}$, slewing platform needs enough starting pressure to be driven from static to movement, but one part of oil comes from pump is used to drive rotary motor, the other changed into the form of energy will be recovered into the accumulator. Therefore, the accumulator pressure increases, the gas volume reduces accordingly. When the motor speed turns to uniform velocity during the time from 6.3 to $14.9 \mathrm{~s}$, both gas pressure and volume in accumulator remain the same. As pilot control reversing valve turns to middle position during the time from 14.9 to 24.9 s, motor is characterized as a pump, and the hydraulic accumulator is full of oil by dynamic torque of rotary platform. Then the accumulator pressure increases and gas volume reduces obviously. When the slewing platform reverse rotation in no-loading condition during the time from 24.9 to 39.9 s, motor rotary is driven by accumulator and pump together. As directional control valve turns to middle position during the time from 39.9 to $49.9 \mathrm{~s}$, motor is characterized as a pump again, and the hydraulic accumulator is also full of oil by dynamic torque of rotary platform.

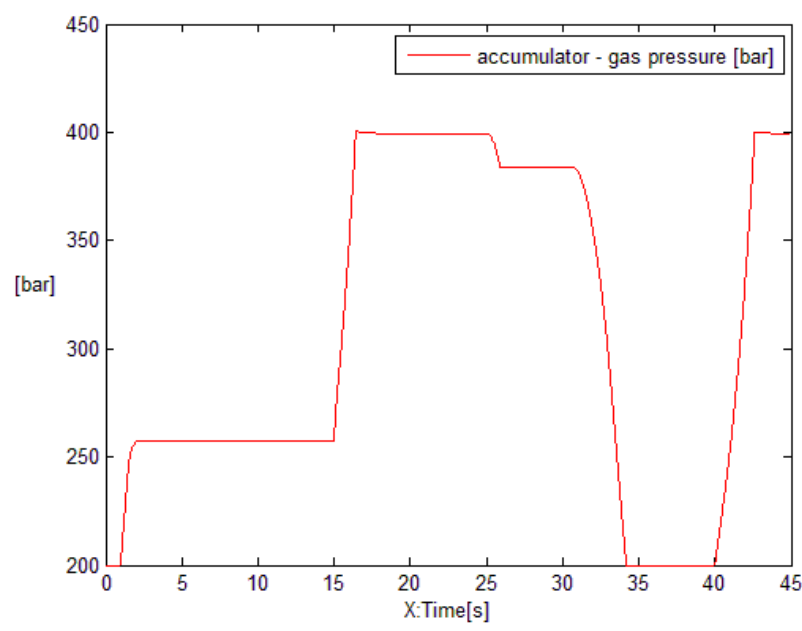

(a) Pressure characteristic curve

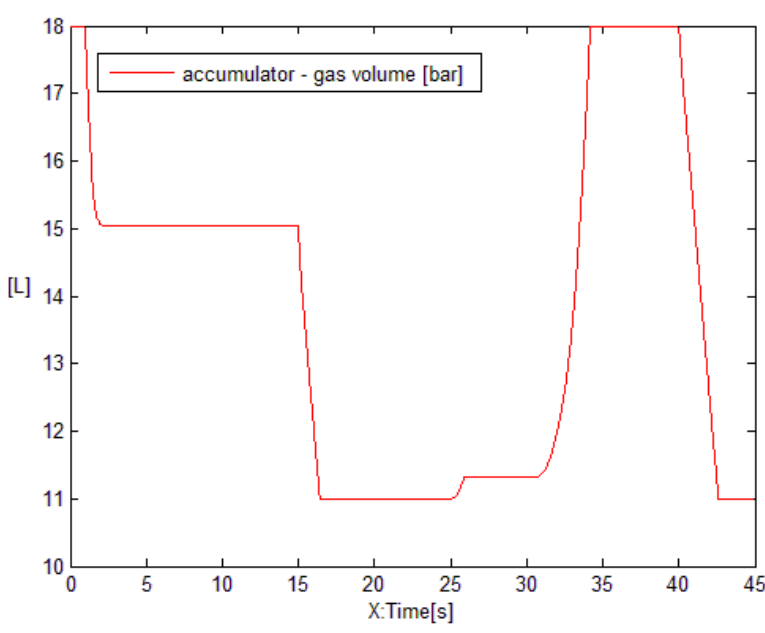

(b) Volume characteristic curve

Fig. 8 Characteristic profile of accumulator in BASS

It exists serious energy waste in open rotary system during braking stage due to excavator inertial platform, so the theoretical loss energy calculation defined as follows: 


$$
\begin{gathered}
E=\frac{1}{2} J \omega_{2}^{2}-\frac{1}{2} J \omega_{b}^{2} \\
E=\frac{1}{2} J \omega_{2}^{2}-0=\frac{1}{2} \times 1 \times 10^{6} \times\left(\frac{8}{60} \times 2 \pi\right)^{2}=350(\mathrm{~kJ})
\end{gathered}
$$

As the calculation above, the energy loss of OCSS is bigger in the process of excavator braking, and then the overflow energy in startup phase is also as high as 350KJ. As shown in Fig. 9, the energy curve of OCSS that whether adopt accumulator shows a trend of increasing, then the pump needs to provide more energy for the system. During the time from 0 to $15 \mathrm{~s}$, the speed of rotation platform increases from 0 to the maximum speed $3934 \mathrm{r} / \mathrm{m}$. The energy curve is rising and rising range in OCSS is more than that in BSSS. During the time from 15 to $25 \mathrm{~s}$, the energy curve tends to straight line and the slewing platform in the braking stage, but the energy is wasted during the period. During the time from 25 to $45 \mathrm{~s}$, the energy curve keeps rising and rising range in OCSS is also larger in comparison to BASS. When the CCSS is in full load or empty load, the energy curve is rising up and down, then rising again. At the 20th s in braking stage, there is a maximum power outlet. After that, energy curve starts to decline rapidly. Through digitization curve and MATLAB calculation, the total outlet power of CCSS is $5032.2 \mathrm{~kJ}$. The total output power of OCSS and BASS is respectively $6327.7 \mathrm{~kJ}$ and $5638 \mathrm{~kJ}$. It is verified that the energy waste more in the stage of starting and braking in OCSS under the same speed. Therefore, the CCSS is more energy efficient than OCSS and BASS.

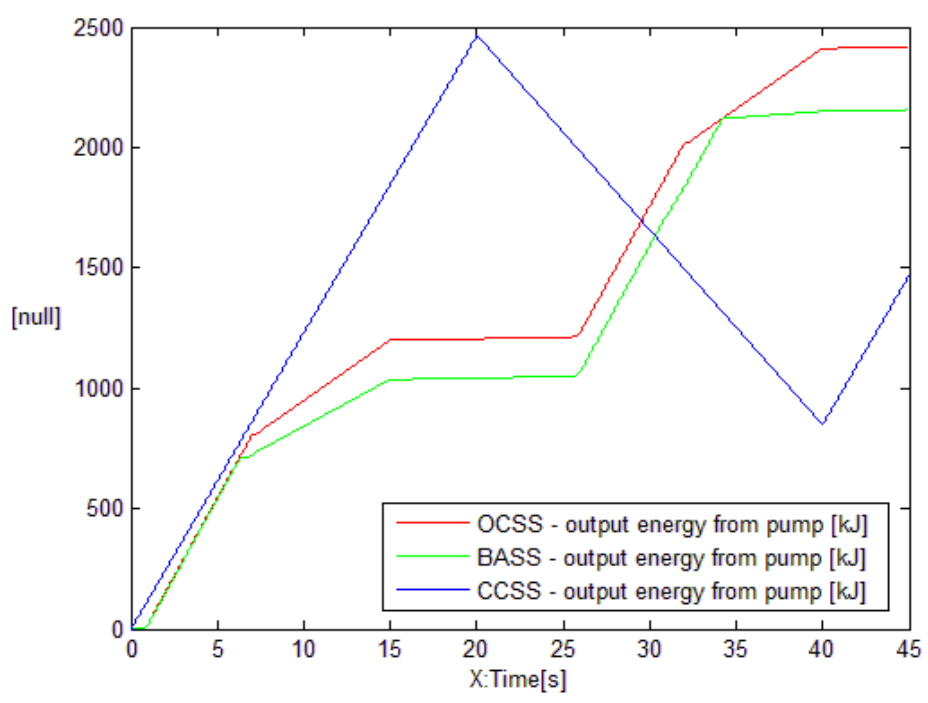

Fig. 9 Energy curve of Hydraulic slewing system

\section{Conclusion}

In this paper, a set of closed-loop control swing circuit is proposed and verified in a hydraulic grasp steel machine that operational weight is 100 tons. Compared with the OCSS and BASS, the motor speed curve of CCSS is almost "double rectangle", which can achieve target speed fast and accurately whatever the loading condition is. Meanwhile, it improves the working efficiency. Moreover, there is not an obvious dithering phenomenon in motor pressure curve. Not only that, the maximum pressure differential generated by pump is smaller and shorter duration in CCSS. That is also benefit to prolong the service life of the whole system, furthermore, making the rotary motion more smooth and stability. Both operating time and energy consumption of swing motion have a great impact on working efficiency, energy conservation and environment protection. So a CCSS is proposed to reduce throttling losses and recuperating regenerative braking energy. Through modeling and simulation, it can be 
concluded that CCSS saves 875.5 KJ and BASS saves 347.2 KJ. So the simulation results show that the swing system with closed-loop control algorithm has better operability than OCSS and BASS and can be better adapted to the typical occasions of the Grasp Steel Machine.

\section{Acknowledgments}

The authors would like to thank the executive editor and the anonymous reviewers for their careful comments and suggestions, which improve the quality of the paper. This work is jointly supported by High-tech Key Research and Development Projects from Science and Technology Department of Sichuan Province, ( Grant No. 2021YFG0056) .

\section{Nomenclature}

$\begin{array}{ll}\text { CCSS } & \text { Closed-loop control swing system } \\ B A S S & \text { Based on accumulator slewing system } \\ \text { OCSS } & \text { Opened-loop control swing system } \\ M & \text { Rotational resistance torque }[\mathrm{N} \cdot \mathrm{m}] \\ F & \text { Working load [N] } \\ C & \text { Wind power coefficient } \\ D & \text { Chord diameter[mm] } \\ L & \text { Length[mm] } \\ X & \text { Center of gravity position } \\ S & \left.\text { Windward area[m }{ }^{2}\right] \\ R & \text { Amplitude[m] } \\ t & \text { Working time[s] }\end{array}$

$\begin{array}{ll}T & \text { Local mean temperature }[\mathrm{K}] \\ U_{i} & \text { Mean velocity components }(\mathrm{i}=1,2,3) \\ \theta, \phi & \left.\text { Rotation angle }{ }^{\circ}\right] \\ \rho & \text { Density of fluid }\left[\mathrm{kg} / \mathrm{m}^{3}\right] \\ \omega & \quad \text { Rotary angular velocity }[\mathrm{r} / \mathrm{min}] \\ J & \text { Rotary inertias }\left[\mathrm{kg} \cdot \mathrm{m}^{2}\right] \\ N & \text { Hydraulic power of slewing mechanism[MPa] } \\ H & \text { Efficiency[\%] } \\ P & \text { Pressure[MPa] } \\ Z & \text { Number of gear tooth } \\ I & \text { Transmission ratio } \\ V & \text { Volume[L] }\end{array}$

\section{References}

[1] Ting C-C, Tsai D-Y, Hsiao C-C. Developing a mechanical roadway system for waste energy capture of vehicles and electric generation. Appl Energy 2012; 92:1-8. https://doi.org/10.1016/j.apenergy.2011.10.006<http://linkinghub.elsevier. com/retrieve/pii/S0306261911006556>.

[2] Hannan M, Azidin F, Mohamed A. Hybrid electric vehicles and their challenges: a review. Renew Sustain Energy Rev 2014; 29:135-50. https://doi.org/10.1016/j.rser.2013.08.097<http://linkinghub.elsevier.com/retrieve/pii/S1364032113006370>.

[3] Huang KD, Tzeng S-C, Ma W-P, Chang W-C. Hybrid pneumatic-power system which recycles exhaust gas of an internal-combustion engine. Appl Energy 2005;82(2):117-32. https://doi.org/10.1016/j.apenergy.2004.10.006<http:// linkinghub.elsevier.com/retrieve/pii/S0306261904001722>.

[4] Doucette RT, McCulloch MD. Modeling the prospects of plug-in hybrid electric vehicles to reduce CO2 emissions. Appl Energy 2011;88(7):2315-23. $\quad$ https://doi.org/10.1016/j.apenergy.2011.01.045<http://www.sciencedirect.com/science/ article/pii/S0306261911000638>.

[5] Xiao. Q, Wang. Q .F, Zhang. Y.T, 2008, “Control strategies of power systemin hybrid hydraulic excavator,” Automation in Construction, Vol.17, No. 4, pp. 361-367. 
[6] Ochiai M, Rye S, 2008, "Hybrid in construction machinery," Proceedings of the 7th JFPS International Symposium on Fluid Power, TOYAMA, Japan, September 15-18, pp. 41-44.

[7] D.Y. Wang, C. Guan, S.X. Pan, M.J. Zhang, X. Lin, 2009, "Performance analysis of hydraulic excavator power train hybridization," Automation in Construction, Vol. 18, No. 3, pp.249-257.

[8] T.L. Lin, Q.F. Wang, B.Z. Hu, et al, 2010, “Development of hybrid powered hydraulic construction machinery,” Automation in Construction, Vol. 19, No. 1, pp.11-19.

[9] A. Hall, 2002, "Characterizing the operation of a large hydraulic excavator Master of Philosophy School of Engineering, University of Queensland,” Brisbane, Australia.

[10] Harr J, 2002, “Arrangement and method relating to a swing damper,” European Patent No. EP1175368.

[11] Dote Y, 1990, “Servo motor and motion control using digital signal processing,” Prentice-Hall, Englewood Cliffs, New Jersey, pp. $97-103$.

[12] Jones B.M, 1974, “Damping circuit for excavator multimotor load sharing swing drive,” US Patent No. 3806780.

[13] Sepehri N, Sassani F, Lawrence D, Ghasempoor A, 1996, "Simulation and experimental studies of gear backlash and stick-slip friction in hydraulic excavator swing motion,” Journal of Dynamic System Measurement \& Control, Vol. 118, No. 3, pp.99-101.

[14] Bin Y, Jiao Z, Douglas K, 1998, "High performance swing velocity tracking control of hydraulic excavators,” Proceedings of the American Control Conference, Philadelphia, Pennsylvania, pp.818-822.

[15] K Jin, T Park, H Lee, 2012, “A control method to suppress the swing vibration of a hybrid excavator using sliding mode approach,” Proceedings of the Institution of Mechanical Engineers, Part C: Journal of Mechanical Engineering Science, Vol.226, pp.1237-1253.

[16] Yu Y-X, Ahn KK. Energy regeneration and reuse of excavator swing system with hydraulic accumulator. Int J Precision Eng ManufactGreen Technol 2020;7(4): 859-73.

[17] Zhang, J.; Ma, P.; Schwab, M. R.; Shang, T.; Patel, K. N., Control system for recovering swing motor kinetic energy. In European Patent: 2011.

[18] Kim Y.J, 2008, "Integrated modeling and hardware-in-the-loop study for systematic evaluation of hydraulic hybrid propulsion options,” Ph. D. Thesis, Department of Mechanical Engineering, University of Michigan, State of Michigan, pp.1-16.

[19] Stienecker A.W, Stuart T, Ashtiani C, 2006, “An ultra capacitor circuit for reducing sulfation in lead acid batteries for mild hybrid electric vehicles," Journal of Power Sources, Vol.156, pp.755-762.

[20] Paladini V, Donateo T, Arturo de Risi AD, Laforgia D, 2007, "Super-capacitors fuel-cell hybrid electric vehicle optimization and control strategy development,” Energy Conversion and Management, Vol.48, pp.3001-3008.

[21] Naoki S, Takao N, Etsujiro I, 2005, “Swing system development of hybrid excavator," Proceedings of Automobile Technology Spring Academic Conference, Vol.36, No.1, pp. 218-222.

[22] Masahiro Miyabe, Akinori Furukawa, Hideaki Maeda, et al, 2009, “A Behavior of the Diffuser Rotating Stall in a Low 
Specific Speed Mixed-Flow Pump,” The International Journal of Fluid Machinery and Systems, Vol.2, No.1, pp.31-39.

[23] H. Yao, Q. F. Wang, 2013, “Control Strategy for Hybrid Excavator Swing System Driven by Electric Motor," IFAC Symposium on Mechatronic Systems, Hangzhou, China, April 10-12, pp.109-115. 